\title{
Protein $v$. carbohydrate intake differentially affects liking- and wanting-related brain signalling
}

\author{
Jurriaan M. Born ${ }^{1,2 *}$, Mieke J. I. Martens ${ }^{1,2}$, Sofie G. T. Lemmens ${ }^{1,2}$, Rainer Goebel ${ }^{3}$ and \\ Margriet S. Westerterp-Plantenga ${ }^{1,2}$ \\ ${ }^{1}$ Top Institute Food and Nutrition, Wageningen, The Netherlands \\ ${ }^{2}$ Department of Human Biology, Maastricht University, Maastricht, PO Box 616, 6200 MD Maastricht, The Netherlands \\ ${ }^{3}$ Department of Cognitive Neuroscience, Maastricht University, Maastricht, The Netherlands
}

(Submitted 18 July 2011 - Final revision received 20 February 2012 - Accepted 20 February 2012 - First published online 29 May 2012 )

\begin{abstract}
Extreme macronutrient intakes possibly lead to different brain signalling. The aim of the present study was to determine the effects of ingesting high-protein $v$. high-carbohydrate food on liking and wanting task-related brain signalling (TRS) and subsequent macronutrient intake. A total of thirty female subjects (21.6 (SD 2.2) years, BMI $25 \cdot 0$ (SD 3.7$) \mathrm{kg} / \mathrm{m}^{2}$ ) completed four functional MRI scans: two fasted and two satiated on two different days. During the scans, subjects rated all food items for liking and wanting, thereby choosing the subsequent meal. The results show that high-protein (PROT) $v$. high-carbohydrate (CARB) conditions were generated using protein or carbohydrate drinks at the first meal. Energy intake and hunger were recorded. PROT (protein: 53.7 (SD 2.1) percentage of energy (En\%); carbohydrate: 6.4 (SD 1.3) En\%) and CARB conditions (protein: 11.8 (SD 0.6) En\%; carbohydrate: 70.0 (SD 2.4) En\%) were achieved during the first meal, while the second meals were not different between the conditions. Hunger, energy intake, and behavioural liking and wanting ratings were decreased after the first meal $(P<0 \cdot 001)$. Comparing the first with the second meal, the macronutrient content changed: carbohydrate $-26.9 \mathrm{En} \%$ in the CARB condition, protein $-37.8 \mathrm{En} \%$ in the PROT condition. After the first meal in the CARB condition, wanting TRS was increased in the hypothalamus. After the first meal in the PROT condition, liking TRS was decreased in the putamen $(P<0.05)$. The change in energy intake from the first to the second meal was inversely related to the change in liking TRS in the striatum and hypothalamus in the CARB condition and positively related in the PROT condition $(P<0.05)$. In conclusion, wanting and liking TRS were affected differentially with a change in carbohydrate or protein intake, underscoring subsequent energy intake and shift in macronutrient composition.
\end{abstract}

\section{Key words: Rewarding value: Liking and wanting: Protein: Carbohydrate: Brain: Functional MRI: Food choices}

The prevalence of obesity and its co-morbidities is increasing in the developed world ${ }^{(1,2)}$. This is caused by energy intake exceeding energy expenditure, i.e. positive energy balance ${ }^{(3)}$, and may be influenced by features of food intake, such as eating in the absence of hunger ${ }^{(4)}$. This overeating is often driven by reward seeking ${ }^{(5)}$. Previous studies ${ }^{(6)}$ suggested that eating palatable food may disrupt food choice, leading to higher energy intake. This increase in food intake was related to BMI and palatability ${ }^{(7)}$. Additionally, evidence was found that glucose intake may bias neurological processes towards higher energy intake ${ }^{(8-10)}$. Conversely, proteins have been shown to assist in weight loss and weight maintenance, by increasing satiety over a meal and decreasing energy intake $^{(11,12)}$. However, compliance to protein diets is relatively low ${ }^{(11)}$, and little is known about the rewarding value of protein and its influence on brain signalling. To study the rewarding value of food, we recently used Berridge's model that describes the rewarding value of food as 'liking', the hedonic preference for, or palatability of a food item, and 'wanting', the motivation to eat a particular food item ${ }^{(13)}$. Energy intake is mainly determined by wanting ${ }^{(5,14)}$, while liking is relatively more stable ${ }^{(14)}$. Additionally, we showed that liking and wanting are signalled in several overlapping as well as specific areas in the human brain ${ }^{(14)}$. We observed that wanting task-related signalling (TRS) was represented significantly in the hypothalamic region and the striatum, and that signalling in the satiated condition in these regions was typically inversely related to $\mathrm{BMI}^{(14)}$; no differences in brain signalling under highcarbohydrate or high-protein conditions have been reported previously. Furthermore, we observed liking TRS in the insula, and when related to cognitive dietary restraint also in the nucleus accumbens ${ }^{(14)}$. Here we assessed the way

Abbreviations: CARB, high carbohydrate; En\%, percentage of energy; fMRI, functional MRI; PROT, high protein; TRS, task-related signalling.

* Corresponding author: J. M. Born, fax +31 433670976, email j.born@maastrichtuniversity.nl 
in which protein $v$. carbohydrate may affect liking- and wanting-related signalling in the human brain in the short term, using functional MRI (fMRI). We hypothesised that protein intake may acutely modulate hypothalamic and striatal signalling differently from carbohydrate intake, thereby differentially affecting brain signalling and motivation for food selection and energy intake in the absence of hunger.

\section{Subjects and methods}

\section{Subjects}

The present study was conducted according to the guidelines laid down in the Declaration of Helsinki and all procedures involving human subjects were approved and registered under MEC 09-3-035 by the Medical Ethical Committee of Maastricht University and at the Netherlands Trial Register under NTR1904. Subject recruitment started on 23 November 2009 and included subjects with BMI $19-33 \mathrm{~kg} / \mathrm{m}^{2}$. A total of thirty-five test subjects were screened and informed, written consent was obtained from all subjects. Inclusion criteria were as follows: healthy female subjects; body weight ranging from normal weight to obese (BMI $19-33 \mathrm{~kg} / \mathrm{m}^{2}$ ); right-handedness. Exclusion criteria were a personal or familial history of psychiatric disorder, recent dieting or the use of intra-uterine contraceptives other than Mirena. Finally, a total of thirty subjects with the following characteristics were included: age 21.6 (SD 2.2) years; BMI $25 \cdot 0\left(\mathrm{SD} 3 \cdot 7 \mathrm{~kg} / \mathrm{m}^{2}\right)$; range $19 \cdot 4-32 \cdot 8 \mathrm{~kg} / \mathrm{m}^{2}$.

\section{Conditions}

For the present study, two conditions were generated using two macronutrient-specific drinks that were designed to be matched for consistency, energy content, taste and pleasantness of taste: a high-carbohydrate (CARB) condition was generated by supplementing the first meal with a drink high in carbohydrate (carbohydrate: 94 percentage of energy (En\%); protein: $6 \mathrm{En} \%$; fat: $0 \mathrm{En} \%)$ and a high-protein (PROT) condition was generated by supplementing the first meal with a drink high in protein (carbohydrate: $10 \mathrm{En} \%$; protein: $87 \mathrm{En} \%$; fat: $3 \mathrm{En} \%$ ). The carbohydrate drink contained (per 100g) $265 \cdot 2 \mathrm{~kJ}$ and consisted of the following: $22.5 \mathrm{~g}$ Optimel yogurt (Friesland Campina); $2.5 \mathrm{~g}$ strawberry syrup (PLUS; PLUS Holding); $10.0 \mathrm{~g}$ Fantomalt (Nutricia); $2.5 \mathrm{~g}$ Nutilis (Nutricia); $0.1 \mathrm{~g}$ Canderel powdered sweetener (Pietercil Barends); $62.5 \mathrm{~g}$ water. The protein drink contained (per $100 \mathrm{~g}$ ) $249.5 \mathrm{~kJ}$ and consisted of the following: $10.0 \mathrm{~g}$ Perfect Protein $85+$ Strawberry (Perfect Body); $2.5 \mathrm{~g}$ Perfect Protein 95 (Perfect Body); $25 \mathrm{~g}$ yogurt ('Magere kwark'; PLUS Holding); $62.5 \mathrm{~g}$ water. The amount of each drink given was individually determined as $14 \%$ of daily energy requirement. Daily energy requirement was estimated using the Harris-Benedict equation to calculate the BMR, and the activity index was assessed using the Baecke questionnaire ${ }^{(15,16)}$. Daily energy requirement was determined as BMR $\times$ activity index. The drinks were given $5 \mathrm{~min}$ before the first meal and had to be consumed completely by the end of the meal.

\section{Functional MRI sessions}

A fasted and satiated condition was created: subjects came fasted to the university. Subjects were instructed not to drink any alcoholic beverages the day before the test and not to eat, or drink coffee or tea after 22.00 hours the evening before testing. Thereby, subjects were fasted for at least $10 \mathrm{~h}$. Adherence to these instructions was individually confirmed. Each session included four visual analogue scale questionnaires for hunger and satiety and two fMRI scans during which the subsequent meal was chosen ${ }^{(14)}$. The visual analogue scale questionnaires consisted of $100 \mathrm{~mm}$ lines, anchored with 'not at all' at the far left and 'extremely' at the far right, and questions asked were 'How hungry are you?' and 'How full do you feel?'. The satiated condition was created by eating the first meal between the first and the second fMRI scan. The second meal followed the second scan. Both meals consisted of the food items that were chosen from thirteen food images within the scanner. Each meal was offered immediately after the scan. The food items that were used had been tasted individually and rated for their taste and texture on a separate day. In addition, the CARB and PROT drinks were tasted and their similarity in hedonic value and texture was individually confirmed. After tasting, all items were known to the subjects ${ }^{(14)}$. The food images were presented against a black background (Fig. 1(a)). All items were resized to match for intensity by the subjects during the tasting session. To account for the limited number of different items, all items were given in large quantities. Subjects were not required to eat all that were offered, but some of each chosen item had to be consumed. The set-up and paradigm during the fasted and satiated state were identical and therefore directly comparable in the analyses.

\section{Functional MRI settings and paradigm}

Subjects were scanned in a Siemens Magnetom Allegra MRI system (Siemens), with the standard one-channel head coil. T1-weighted scout images were used to confirm the subjects' position. During the functional run, four segments of wanting and four segments of liking were presented. The food images were shown one by one in an event-related design (Fig. 1(a) and (b)), thus presented one by one and the subjects were asked to rate each for 'liking' and 'wanting, ${ }^{\text {(14) }}$. Berridge's model for the rewarding value of food was used, i.e. liking and wanting ${ }^{(13)}$. Thus, behavioural liking and wanting were assessed using the ratings obtained in the scanner. Here, the question to determine liking was 'How much do you like this item, not considering if you want to eat it right now?'. Wanting was determined using the question 'How much do you want to eat this item right now?', while the subjects were instructed that the items that were rated high in wanting had to be consumed immediately after the scan. 'Liking' thereby represented explicit food preference and 'wanting' represented explicit food choice. In total, the functional run took approximately $35 \mathrm{~min}$ and each image was shown four to five times. A four-button response box was used to obtain the ratings (LUMItouch; Photon Control) using the right hand. The buttons represented values of 1-4. The average ratings were calculated directly after the scan 


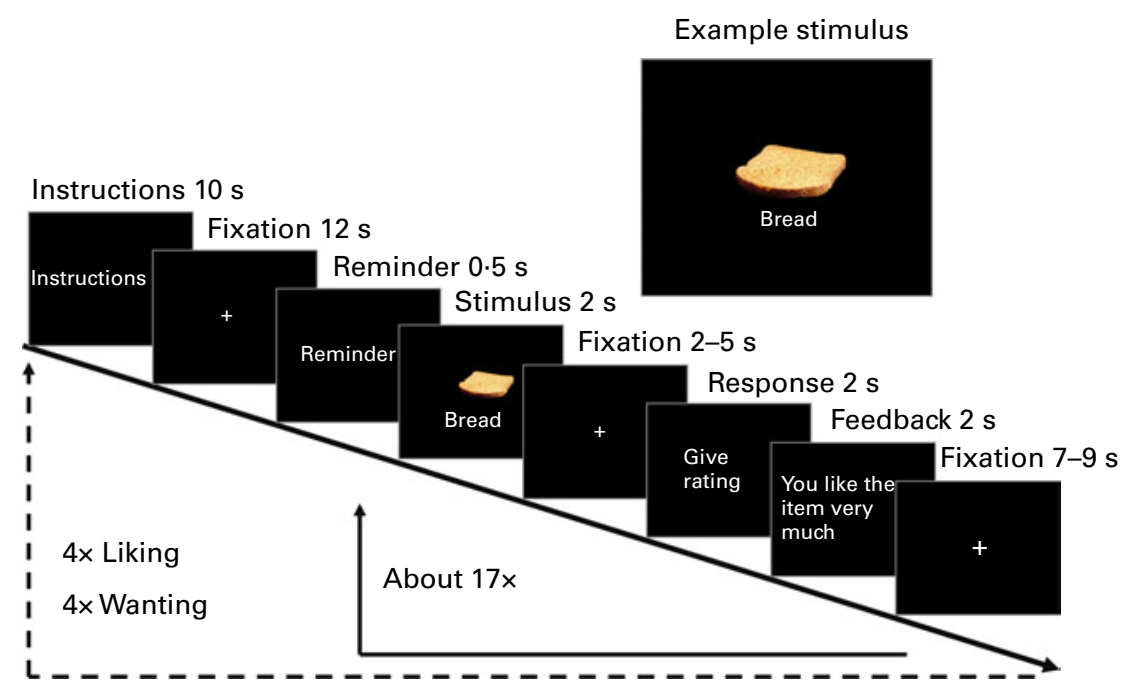

Fig. 1. An overview of the functional MRI (fMRI) paradigm. Each fMRI session took approximately 45 min including a 35 min fMRI run. (a) Stimuli slide layout: each food image was shown centred with the food name below the image. Images were standardised for size and colour. (b) Four segments of each liking and wanting were shown (dotted line) in counterbalanced order. Segments contained approximately seventeen event-related stimulus trials (solid line).

and items rated 2.5 or higher were offered to eat. fMRI images were acquired throughout the session using a T2*-weighted protocol to obtain a blood oxygen level-dependent T2* signal (repitition time (TR) $2 \mathrm{~s}$, echo time (TE) $26 \mathrm{~ms}$, flip angle $90^{\circ}$, matrix $96 \times 96$, field of view 269, voxel size $3.0 \times 3.0 \times 2.9 \mathrm{~mm}$, gap $0.1 \mathrm{~mm}$ resulting in a functional voxel size of $27 \mathrm{~mm}^{3}$ ).

\section{Functional MRI data preprocessing}

All fMRI data were imported, aligned and analysed using BrainVoyager QX version 2.3 (Brain Innovation B.V.). Preprocessing was performed using the standard methods in BrainVoyager $\mathrm{QX}^{(14)}$. To align all functional images, auto-alignment (sixparameter affine) to each subjects' own $1 \mathrm{~mm}$ isovoxel highresolution T1-weighted anatomical scan was performed and all images were transformed into the Talairach coordinate system $^{(17)}$. The resulting resolution was $3 \times 3 \times 3 \mathrm{~mm}$, i.e. a functional voxel size of $27 \mathrm{~mm}^{3}$.

\section{Region of interest definition}

Anatomical regions of interest related to our hypotheses were determined from our earlier study and the regions of interest definition files from that study were used ${ }^{(14)}$. Exploratory analyses were used to identify regions to be included and included regions were bilateral caudate, nucleus accumbens, putamen and hypothalamic region.

\section{Behavioural measurements}

Visual analogue scales for hunger and satiety were taken four times to determine the effects of a pre-meal (hunger) and a state after a meal (absence of hunger). These questionnaires were obtained before and after each scan. Thus, the first meal was eaten in-between questionnaires 2 and 3, the second meal was eaten after questionnaire 4. In addition, the liking and wanting ratings that were given for each item were recorded and analysed.

\section{Data analysis}

Brain activation was analysed using a general linear model approach with separate predictors for liking and wanting. Using the subjects' image ratings, each image was ranked according to its average rating. The five highest and five lowest images were selected to avoid an unbalanced design. Consequently, the predictors obtained were 'low liking', 'high liking', 'low wanting' and 'high wanting'. Each trial was defined as the duration of the image presentation and was modelled using the standard canonical two- $\gamma$ haemodynamic response function and additional predictors were created to contain the nonselected trials. Furthermore, several confounding predictors were included: motion estimates obtained in the motion correction step of functional data preprocessing were de-trended, $z$-normalised and high-pass filtered (cut-off $0.025 \mathrm{~Hz}$ ) using the BrainVoyager Analysis-Predictor tool (BVA-Predictor Tool, J. M. Born), and subsequently, a multi-subject general linear model was run using these transformed motion estimates on the subjects' data. The three strongest predictors (group average) were included: $y$ and $z$ translation and $x$ rotation. Linear habituation was modelled as a single predictor from all model parameters (BVA-Predictor Tool). Additionally, onsets of instructions, reminder and response request were modelled using the two- $\gamma$ haemodynamic response function to account for the variation associated with these visual stimuli. Dummy coding was used to extract group effects for pre-meal and after the first meal and CARB $v$. PROT conditions (using the BVAPredictor Tool). From regions of interest, parameter estimates for the wanting task-related models were extracted and baseline corrected, in which baseline represents the parameter estimates for the constant in the general linear model. All data were 
analysed using Microsoft Excel (Microsoft Corporation, Redmond, WA, USA) and PASW statistics 18 (IBM Corporation, Somers, NY, USA).

\section{Results}

\section{Hunger and satiety}

The hungry $v$. satiated condition was confirmed by high ratings for hunger pre-meal (71.5 ( $\mathrm{SD} 3.8) \mathrm{mm}$ visual analogue scale) and low ratings after the first meal (15.3 (SD 2.7), difference $P<0.001)$ in both conditions. No significant differences were observed related to BMI.

\section{Energy intake and macronutrient composition of the meals}

Comparing the first meal and the second meal, energy intake was significantly lower during the second meal $(P<0 \cdot 001$; Table 1). In the PROT condition, the macronutrient composition was significantly different compared with the CARB condition $(P<0 \cdot 001$; Table 1$)$. Fat intake was not significantly different comparing the conditions. During the second meal, the macronutrient composition of the meals was not significantly different comparing the CARB with the PROT condition (Table 1). Energy intake was not significantly different between the CARB $v$. PROT conditions. The reduction in energy intake implied a relative reduction of carbohydrate intake in the CARB condition from 69.9 to $43.0 \mathrm{En} \%$ and a relative reduction of protein in the PROT condition from 54.7 to $16.9 \mathrm{En} \%$, thereby showing a condition-specific change in macronutrient composition.

\section{Liking and wanting in the carbohydrate v. protein condition}

Behavioural liking and wanting ratings were lower after the first meal $(P<0 \cdot 001)$, but the change in wanting was of seven times higher magnitude (liking: -0.09 (SD 0.14), wanting: -0.72 (SD 0.47), liking $v$. wanting $P<0.001$ ).

In the putamen, liking TRS was reduced post-meal in the PROT condition, but not in the CARB condition (interaction effect, protein $<$ carbohydrate, $P<0.03$; Fig. 2(a)). A similar trend was found for wanting TRS after the first meal in the hypothalamic region (interaction effect, protein $<$ carbohydrate, $P=0.067$; Fig. 2(b)).

When relating the change in energy intake from the first meal to the second meal, no significant relationships between energy intake and wanting signalling were found. Conversely, the change in liking signalling was inversely related to the change in energy intake from pre-meal to post-meal in the CARB conditions, in the hypothalamic region and putamen $(P=0.02$ and 0.08 ; Fig. 2(c) and (d)), while these parameters exhibited a positive correlation in the PROT conditions, in the hypothalamic region $(P=0.03$; Fig. 2(c)). This difference in brain signalling between the PROT and CARB conditions was significant in both regions $(P<0.01)$. Including BMI in the models did not show a significant effect of BMI on liking and wanting TRS related to the macronutrient intakes.

\section{Discussion}

High hunger ratings pre-meal and low hunger ratings after the first meal and a significantly decreased energy intake during the second meal compared with the first meal confirmed the hungry $v$. satiated state, while significantly different macronutrient composition confirmed the CARB $v$. PROT conditions during the first meal. During the second meal, the macronutrient composition was not different between the conditions, thus a condition-specific change occurred from the first meal to the second meal. Behavioural liking and wanting were not different between the CARB and PROT conditions and were not influenced by the subjects' BMI. Both decreased significantly from before to after the first meal, while confirming the higher relative stability of liking compared with wanting: the decrease in wanting was of seven times higher magnitude. This is in line with expectations about liking and wanting as well as earlier findings that indicated strong decreases in behavioural wanting over a meal consumption while changes in liking were either absent or relatively small ${ }^{(14,18-20)}$. Previously, we have shown that liking and wanting are affected differently by different food items (cottage cheese $v$. chocolate mousse) that were liked significantly differently ${ }^{(18)}$. When the differences between the food items are limited to the macronutrient composition, such as in the present study, the subsequent changes in behavioural liking and wanting are not affected differently.

Table 1. Macronutrient composition and energy content of the consumed meals (Mean values with their standard errors)

\begin{tabular}{|c|c|c|c|c|c|c|c|c|}
\hline & \multicolumn{4}{|c|}{ CARB condition } & \multicolumn{4}{|c|}{ PROT condition } \\
\hline & \multicolumn{2}{|c|}{ First meal } & \multicolumn{2}{|c|}{ Second meal } & \multicolumn{2}{|c|}{ First meal } & \multicolumn{2}{|c|}{ Second meal } \\
\hline & Mean & SEM & Mean & SEM & Mean & SEM & Mean & SEM \\
\hline Carbohydrate (En\%) & $69.9^{*}$ & $2 \cdot 0$ & 43.0 & $6 \cdot 0$ & $25 \cdot 3$ & 1.2 & $52 \cdot 1$ & 5.5 \\
\hline Protein $(\mathrm{En} \%)$ & $12 \cdot 0^{\star}$ & 0.6 & $18 \cdot 6$ & 0.3 & $54 \cdot 7$ & $2 \cdot 0$ & $16 \cdot 9$ & 4.6 \\
\hline Fat $(E n \%)$ & $18 \cdot 1$ & 1.8 & 38.4 & $5 \cdot 1$ & $19 \cdot 9$ & 1.5 & 30.9 & 4.6 \\
\hline Energy (kJ) & $2536 t$ & 120 & 264 & 62 & $2349+$ & 21 & 303 & 12 \\
\hline
\end{tabular}

CARB, high carbohydrate; PROT, high protein; En\%, percentage of energy.

${ }^{*}$ Mean values were significantly different for the CARB condition from that of the PROT condition $(P<0.001)$.

$\dagger$ Mean values were significantly different for the first meal $v$. second meal $(P<0.001)$. 

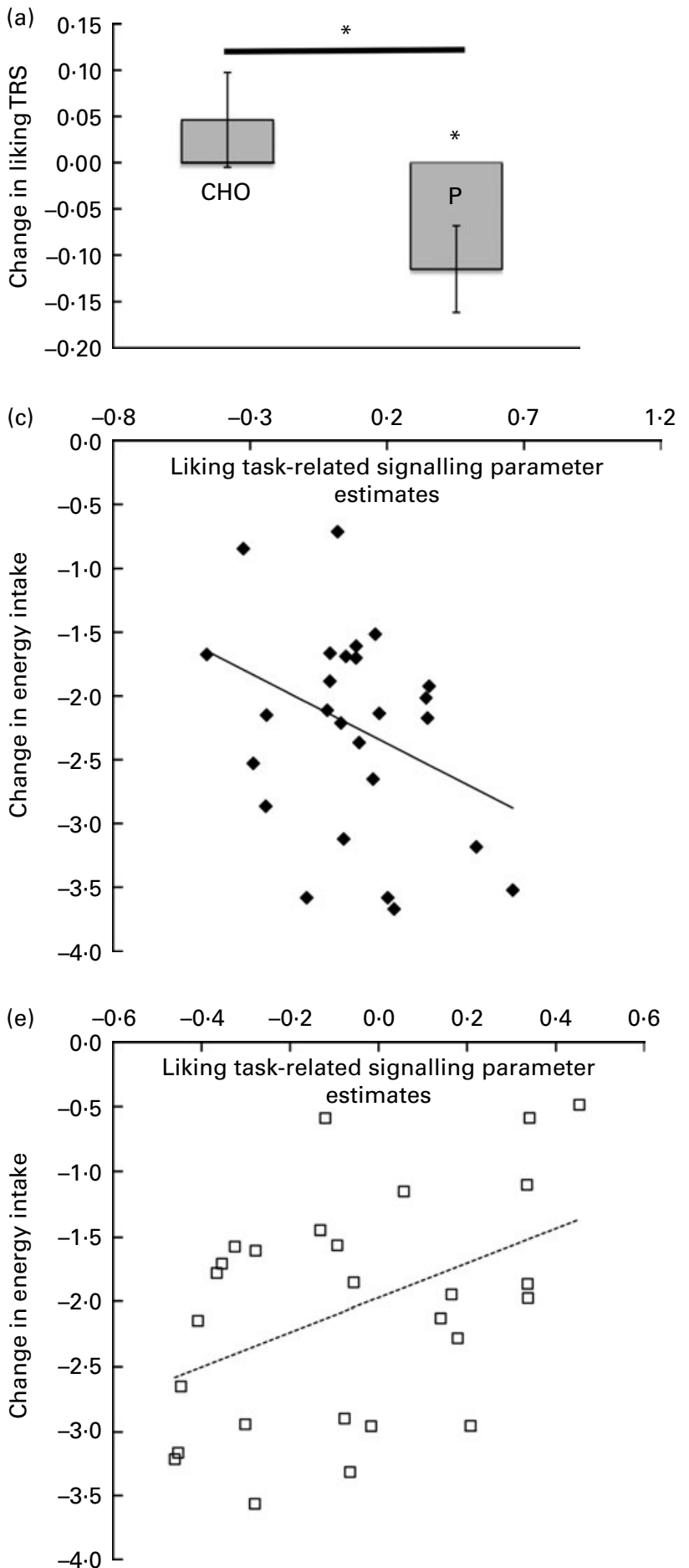

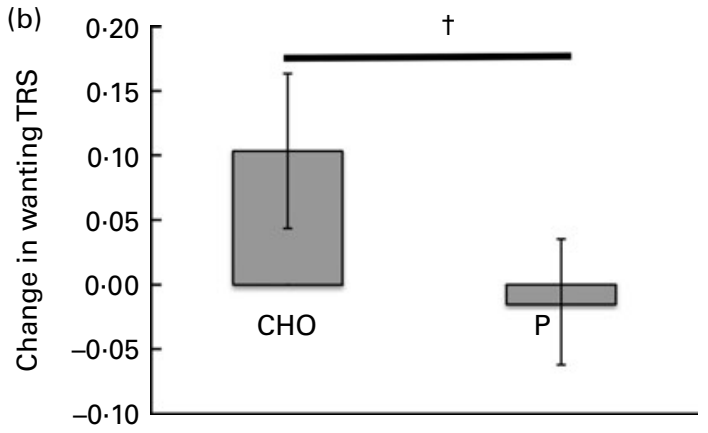

(d)
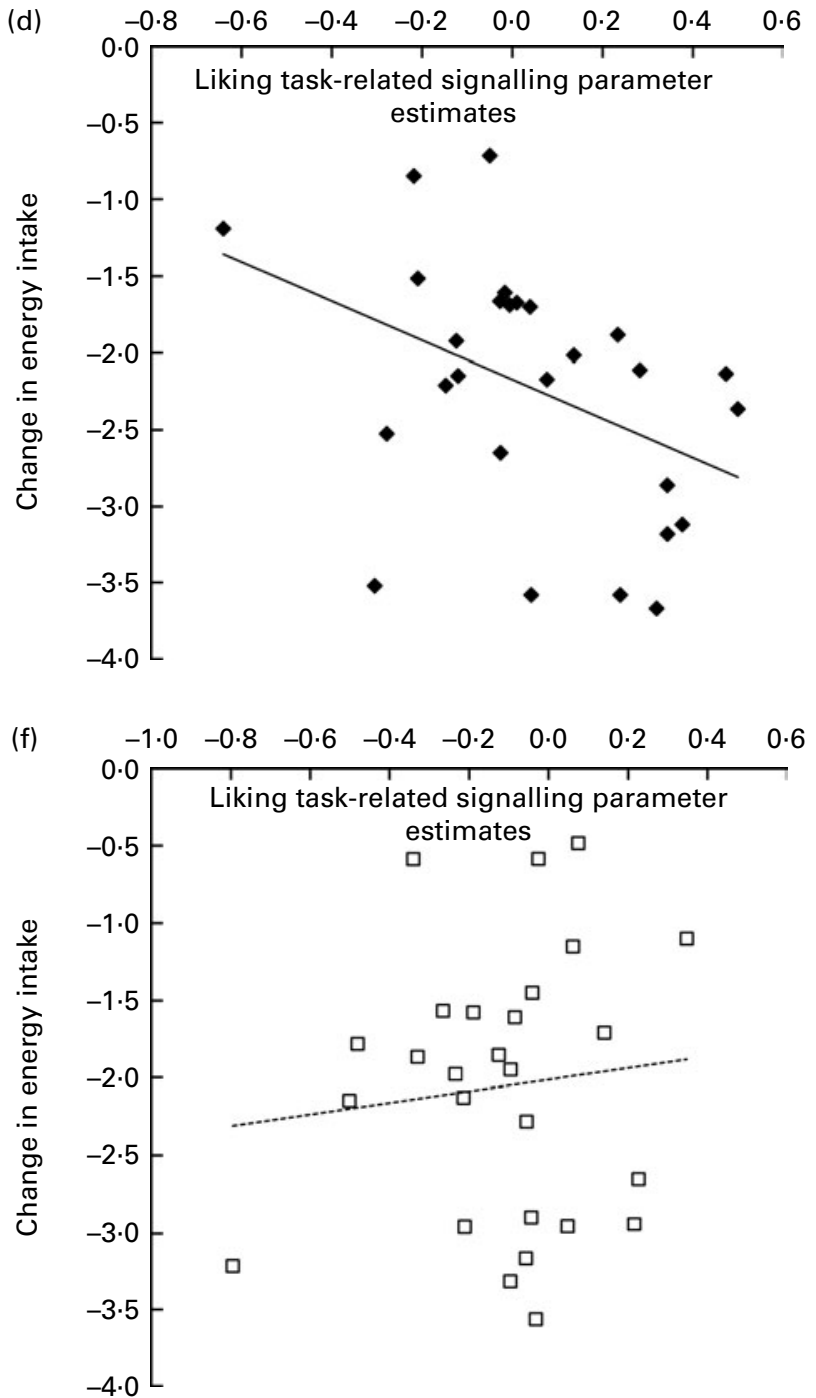

Fig. 2. Functional data from the putamen and hypothalamic regions. (a) Change in liking task-related signalling (TRS) over the meal in the putamen over the meal, with a significant interaction effect of meal $\times$ condition, ${ }^{*} P=0.025$. (b) Change in wanting TRS over the meal in the hypothalamic region, with a trend towards the interaction effect of meal $\times$ condition, $\dagger P=0.067$. Values are means, with their standard errors represented by vertical bars. The change in liking TRS related to the change in energy intake from breakfast to the second meal, in the carbohydrate condition in (c) the putamen $(P<0.04)$ and $(d)$ the hypothalamic region $(P<0.09)$ and in the protein condition in (e) the putamen (NS) and (f) the hypothalamic region $(P<0.04)$. CHO, carbohydrate; $P$, protein.

In the brain, liking TRS in the putamen showed an interaction effect of meal and condition, such that it was reduced after the first meal in the PROT condition while it did not change in the CARB condition. The decrease in energy intake, containing a relative decrease in protein intake from the first meal to the second meal, was positively related to the decrease in liking TRS in the PROT condition, while it was overall negatively related in the CARB condition in the putamen and hypothalamic region. Additionally, liking TRS was higher in the PROT condition compared with the CARB condition, after the first meal 
compared with pre-meal in the putamen, and this was underscored by a similar trend of wanting TRS in the hypothalamus. Thereby, liking TRS exhibited significant opposite directionality comparing the CARB $v$. PROT conditions, without leading to a differential behavioural effect in the short term. Additionally, previous studies have shown both increased and decreased signalling associated with motivation to eat ${ }^{(17,21-24)}$.

In the present study, the different directionality of liking TRS in the putamen and hypothalamic region may underscore the different changes of macronutrient intakes from the first meal to the second meal, both resulting in similar macronutrient intakes during the second meal. Therefore, liking TRS might represent the relative change in food choice at the following meal. While in previous studies BMI was observed to typically affect wanting TRS in several regions ${ }^{(14)}$, no effect was observed on liking and wanting TRS related to protein or carbohydrate intake and the subsequent change to normal macronutrient composition. This may indicate that BMI is related to quantitative rather than qualitative aspects of food intake.

In summary, the present study showed that after a carbohydrate-rich meal, but not after a protein-rich meal, wanting TRS was increased, which may potentially stimulate energy intake. Finally, the increase in liking TRS after a carbohydraterich meal was inversely related to the decrease in energy intake. This underscored especially the relative decrease in carbohydrate intake. Similarly, the decrease in liking TRS after a protein-rich meal positively related to the decrease in energy intake, underscoring the relative decrease in protein intake.

In conclusion, wanting and liking TRS were affected differentially after carbohydrate- or protein-rich meals, underscoring subsequent energy intake and change in macronutrient composition.

\section{Acknowledgements}

This study was funded by the Top Institute Food and Nutrition (TIFN), Wageningen, The Netherlands. The TIFN did not influence the study design, implementation, analysis or interpretation of the data. The authors' contributions are as follows: J. M. B. and M. S. W.-P. designed the study; R. G. and S. G. T. L. advised on the set-up of the experiment; J. M. B. conducted the research; M. J. I. M. and S. G. T. L. assisted during the research; J. M. B. and M. S. W.-P. analysed the data; R. G. advised regarding the fMRI analysis; J. M. B. and M. S. W.-P. wrote the manuscript; J. M. B. had primary responsibility for the final content. All authors read and approved the final manuscript. The authors declare that they have no conflict of interest.

\section{References}

1. Seidell JC (2006) Epidemiology and health economics of obesity. Medicine 34, 506-509.

2. Catenacci VA, Hill JO \& Wyatt HR (2009) The obesity epidemic. Clin Chest Med 30, 415-444.

3. Westerterp KR \& Speakman JR (2008) Physical activity energy expenditure has not declined since the 1980s and matches energy expenditures of wild mammals. Int $J$ Obes 32, 1256-1263.
4. Rutters F, Nieuwenhuizen AG, Lemmens SGT, et al. (2009) Acute stress-related changes in eating in the absence of hunger. Obesity 17, 72-72.

5. Epstein LH, Leddy JJ, Temple JL, et al. (2007) Food reinforcement and eating: a multilevel analysis. Psychol Bull 133, 884-906.

6. Erlanson-Albertsson C (2005) How palatable food disrupts appetite regulation? Basic Clin Pharmacol Toxicol 97, $61-73$.

7. Beaver JD, Lawrence AD, van Ditzhuijzen J, et al. (2006) Individual differences in reward drive predict neural responses to images of food. J Neurosci 26, 5160-5166.

8. Chambers ES, Bridge MW \& Jones DA (2009) Carbohydrate sensing in the human mouth: effects on exercise performance and brain activity. J Physiol 587, Pt 8, 1779-1794.

9. Drewnowski A, Krahn DD, Demitrack MA, et al. (1992) Taste responses and preferences for sweet high-fat foods: evidence for opioid involvement. Physiol Behav 51, $371-379$.

10. Melanson KJ, Westerterp-Plantenga MS, Saris WHM, et al. (1999) Blood glucose patterns and appetite in time-blinded humans: carbohydrate versus fat. Am J Physiol Regul Integr Comp Physiol 277, R337-R345.

11. Soenen S \& Westerterp-Plantenga MS (2008) Proteins and satiety: implications for weight management. Curr Opin Clin Nutr Metab Care 11, 747-751.

12. Veldhorst M, Smeets A, Soenen S, et al. (2008) Protein-induced satiety: effects and mechanisms of different proteins. Physiol Behav 94, 300-307.

13. Berridge KC (1996) Food reward: brain substrates of wanting and liking. Neurosci Biobehav Rev 20, 1-25.

14. Born JM, Lemmens SGT, Formisano E, et al. (2011) Differences between liking and wanting signals in the human brain and relations with cognitive dietary restraint and body mass index. Am J Clin Nutr 94, 392-403.

15. Harris JA \& Benedict FG (1918) A biometric study of human basal metabolism. Proc Natl Acad Sci U S A $\mathbf{4}$, $370-373$.

16. Baecke J, Burema J \& Frijters J (1982) A short questionnaire for the measurement of habitual physical activity in epidemiological studies. Am J Clin Nutr 36, 936-942.

17. Talairach J \& Tournoux P (1988) Co-planar Stereotaxic Atlas of the Human Brain. Stuttgart: Thieme.

18. Born JM, Lemmens SGT, Rutters F, et al. (2010) Acute stress and food-related reward activation in the brain during food choice during eating in the absence of hunger. Int $J$ Obes 34, 172-181.

19. Lemmens SGT, Schoffelen PFM, Wouters L, et al. (2009) Eating what you like induces a stronger decrease of 'wanting' to eat. Physiol Behav 98, 318-325.

20. Lemmens SG, Rutters F, Born JM, et al. (2010) Stress augments food 'wanting' and energy intake in visceral overweight subjects in the absence of hunger. Physiol Behav 103, 157-163.

21. Davis C, Patte K, Levitan R, et al. (2007) From motivation to behaviour: a model of reward sensitivity, overeating, and food preferences in the risk profile for obesity. Appetite 48, 12-19.

22. Davis C, Strachan S \& Berkson M (2004) Sensitivity to reward: implications for overeating and overweight. Appetite 42, 131-138.

23. Franken IH \& Muris P (2005) Individual differences in reward sensitivity are related to food craving and relative body weight in healthy women. Appetite 45, 198-201.

24. Wang GJ, Volkow ND, Logan J, et al. (2001) Brain dopamine and obesity. Lancet 357, 354-357. 